\title{
BMJ Open Reasoning like a doctor or like a nurse? An integrative review protocol
}

\author{
Jettie Vreugdenhil (D , ${ }^{1}$ Sunia Somra, ${ }^{1}$ Hans Ket, ${ }^{2}$ Eugène J F M Custers, ${ }^{3}$ \\ Marcel E Reinders, ${ }^{4}$ Jos Dobber, ${ }^{5}$ Rashmi A Kusurkar ${ }^{1}$
}

To cite: Vreugdenhil J, Somra S, Ket H, et al. Reasoning like a doctor or like a nurse? An integrative review protocol. BMJ Open 2021;11:e049862. doi:10.1136/ bmjopen-2021-049862

- Prepublication history and additional supplemental material for this paper are available online. To view these files, please visit the journal online (http://dx.doi.org/10.1136/ bmjopen-2021-049862).

Received 12 February 2021 Accepted 13 September 2021

Check for updates

(C) Author(s) (or their employer(s)) 2021. Re-use permitted under CC BY-NC. No commercial re-use. See rights and permissions. Published by BMJ.

${ }^{1}$ Amsterdam UMC, Research in Education, Faculty of Medicine Vrije Universiteit Amsterdam, Amsterdam, The Netherlands

${ }^{2}$ Medical Library, Vrije

Universiteit Amsterdam, Amsterdam, The Netherlands

${ }^{3}$ University Medical Centre,

Utrecht University, Utrecht, The

Netherlands

${ }^{4}$ Amsterdam UMC, Student Education Family Medicine,

Faculty of Medicine, Vrije

Universiteit Amsterdam,

Amsterdam, The Netherlands

${ }^{5}$ Amsterdam School of Nursing,

Amsterdam University of Applied

Sciences, Amsterdam, The

Netherlands

Correspondence to MSc Jettie Vreugdenhil; cj.vreugdenhil@amsterdamumc. $\mathrm{nl}$

\begin{abstract}
Introduction Clinical reasoning, a major competency for all health professionals, has been defined and studied 'within' each profession. We do not know if content, process and outcomes are comparable 'between' physician and nursing clinical reasoning. This paper aims to set up a protocol for an integrative review to analyse and synthesise the scientific nursing and medical clinical reasoning literature. It builds on the history of nursing and medical clinical reasoning research and aims to create a higher level of conceptual clarity of clinical reasoning, to increase mutual understanding in collaboration in patient care, education and research.
\end{abstract}

Methods and analysis This integrative review follows stepwise the methods described by Whittmore and Knafl: problem identification, literature search, data evaluation, data analysis and presentation.

The initial systematic and comprehensive search strategy is developed in collaboration with the clinical librarian and is performed in electronic databases, PubMed, CINAHL, Psyclnfo and Web of Science from 30 March 2020 to 27 May 2020. Empirical and theoretical studies are included. This search will be accompanied by ancestry searching and purposeful sampling. A Preferred Reporting Items for Systematic Reviews and Meta-Analyses flow chart will summarise the selection process. The quality of eligible studies will be evaluated with a checklist, suitable for diverse study methods.

The data analysis is inspired by concept analysis of Walker and Avant and layered analysis of an intervention of Cianciolo and Regehr. We will extract the data of the included studies conforming these layers and features, to capture the multifaceted nature of clinical reasoning in both professions. The data will be presented in a validity matrix to facilitate comparing and contrasting.

Ethics and dissemination Ethics approval is not required. The outcomes will be disseminated through conference presentations and publications.

\section{INTRODUCTION}

Clinical reasoning is an essential competency for all health professionals. Clinical reasoning has always been defined, practised, researched, taught and guided 'within' the boundaries of each health profession. Within and between professions, differences exist in terminology and connotations. ${ }^{1}$ However, all clinical reasoning starts with
Strengths and limitations of this study

This study will be the first to systematically investigate presumed differences between clinical reasoning of physicians and nurses.

- With a rigorous and stepwise approach, the whole spectrum of scientific publications on clinical reasoning in nursing and medicine, including qualitative, quantitative and theoretical, will be identified, screened and selected for analysis.

- The data extraction and synthesis will be conducted using three analyses types: concept analysis, layered analysis and simultaneous concept analysis. It is expected to identify and explain the similarities and differences in reasoning.

- A limitation of this integrative review is the high number of publications on the topic and the use of many (surrogate) terms, related to clinical reasoning.

the meeting of a patient and his experiences. As an example, all health professionals encounter patients complaining of tiredness or exhaustion. From the patients' perspectives, fatigue disrupts their quality of life. ${ }^{2}$ From the health professionals' perspectives, fatigue could be a symptom, a state, a diagnosis, a rationale for therapy, a side-effect of treatment, a result of undertraining or overtraining, or an important prognostic factor in a patient management plan, depending on which health profession one belongs to. Thus, fatigue has a different position in the professional reasoning approaches of therapists, physicians and nurses.

\section{Background}

Clinical reasoning, which is a highly complex system with multiple interdependent mental activities, ${ }^{3}$ can be viewed from three perspectives: the process of reasoning, the knowledge structures, and the extent of analytical or intuitive cognitive modes. ${ }^{4}$ Clinical reasoning is considered a multifaceted concept, coupled with the potential for misunderstanding. ${ }^{5}$ In the clinical reasoning literature, concepts or terms like clinical judgement, 
decision-making, and critical thinking have been used as surrogate or related terms or as synonyms. ${ }^{15-7}$

According to previous studies, differences in terminology, operationalisations, understanding and perspectives may hamper three domains: education and assessment, ${ }^{58}$ collaboration and communication, ${ }^{9}{ }^{10}$ and research. ${ }^{11}$

In education and assessment, Young et $a \tilde{l}$ and Brown Tyo and McCurry ${ }^{8}$ state that the inconsistency in conceptualisation and terminology is a barrier in identifying effective teaching and assessment strategies. We learn from Muller-Juge et $a t^{\theta}$ and Visser $e t a l^{10}$ about interprofessional collaboration, that residents and nurses have different perceptions and expectations about clinical reasoning of the other professional. ${ }^{9}$ Students of different professions could not fully understand their patients' condition, if they were not aware of the reasoning (process and outcomes) of their colleagues. ${ }^{10}$ In research, in a scoping review, clinical reasoning has been investigated as a cognitive, contextually situated or socially mediated activity, reflecting the multidimensionality of clinical reasoning. ${ }^{11}$ From the existing literature, this review was triggered by: the multifaceted characteristic of clinical reasoning and the lack of awareness of the clinical reasoning of other professionals in interprofessional teams.

\section{Rationale}

Hence, even though clinical reasoning has been extensively investigated, this has typically been carried out within a profession, while less attention has been paid to the differences and similarities between professions, like between medicine and nursing.

Since most research on clinical reasoning has been conducted in the medical profession, we also need to know if, and to what extent, these results can be generalised to the nursing domain. For example, both professions share an educational research tradition in studying the differences between novices and experts. However, Chiffi and Zanotti ${ }^{12}$ state that medicine is concerned with biofunctional alterations of a patient, and that nursing is focused on independence in self-care and well-being.

Assuming that this is understood correctly, and that we do not know whether content, process and outcomes of clinical reasoning of physicians and nurses are comparable, this also creates uncertainty about the mutual transferability of research findings. If we could succeed to unravel and describe the differences and similarities between nurses' and physicians' reasoning, we may also learn more about the features of clinical reasoning in general and its impact on collaboration in patient care and research. Making clinical reasoning more explicit may facilitate understanding among physicians and nurses and improve teaching, guidance and assessment of reasoning. ${ }^{58}$ These possibilities call for further inquiry into clinical reasoning from both professional perspectives. To our knowledge, few comparative studies have been published and no systematic review of similarities and differences in reasoning.

\section{Objectives}

Because clinical reasoning is assumed to be a multifaceted concept, this investigation will have to consider all these facets, properties and relations, with the aim to 'peel the shells from this onion'.

We identified two methods of analysis for our study. Cianciolo and Regehr have described the layers of an educational intervention: philosophy, principles, techniques and contextual influences, ${ }^{13}$ and Walker and Avant have described the method of concept analysis. A concept is a mental construction and contains attributes, borders, antecedents and consequences. ${ }^{14}$ Therefore, through the lens of layers and concepts, our research questions are: what are the features of clinical reasoning of professional practitioners as described in medical and nursing scientific literature and what can we learn from this simultaneous concept analysis? Our broader aim is to create a higher level of conceptual clarity of clinical reasoning of nurses and physicians, to increase mutual understanding in collaboration in patient care, education and research.

\section{METHODS AND ANALYSIS}

To answer our research question, we chose and will apply the integrative review method. The purpose of this method is to summarise and to critically analyse what is known about concepts, theories or methodologies, in order to report the current state of evidence and to identify future goals for research and practice. An integrative review has a broader research question than a systematic review, and follows a systematic, comprehensive, stepwise approach to increase the understanding of a phenomenon. ${ }^{15}$ Moreover, this method allows to include and combine empirical qualitative and quantitative studies and theoretical articles like all types of reviews, to collect the whole spectrum of perspectives on the topic. ${ }^{16} 17$ The integrative review method fits our aim and the rich, diverse literature on clinical reasoning.

\section{Patient and public involvement}

In this study, patients, students and educators are included only through inclusion of what is written about them in the published studies.

The review process follows five stages, as shown in figure $1 .^{16}$

\section{Problem identification}

As described in the introduction, to alleviate the lack of explicit knowledge about how the clinical reasoning of one profession relates to that of the other may help us to improve interprofessional collaboration, education and research.

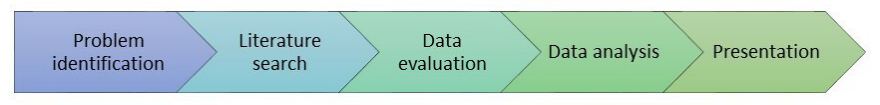

Figure 1 Stages of an integrative review (Whittmore and Knafl ${ }^{16}$ ). 


\section{Literature search}

The use of more than one literature search procedure is recommended when conducting an integrative review. ${ }^{16}$ We planned two procedures to search: a systematic, comprehensive search strategy, followed by ancestry searching. This involves reviewing the references of included studies from the first procedure. ${ }^{18}$ Initially, the main researcher and a clinical librarian (JCV, HK) searched comprehensively, from 30 March 2020 to 27 May 2020, from inception to date in PubMed, CINAHL, PsycInfo and Web of Science, with the search terms clinical reasoning, clinical decision making, clinical judg(e) ment, collaborative reasoning, diagnostic reasoning, reasoning inductive, deductive, inference, probability judgment of nurses and physicians (all specialties). The search terms were chosen mainly on the studies of Young et $a \tilde{e}$ and Victor-Chmil, because they provide and explain the terminology used in clinical reasoning publications. ${ }^{6}$ A worked out search string is included in online supplemental appendix 1 .

As a third procedure, if the yield of the number of articles is too large to analyse, purposeful sampling can be considered. ${ }^{16}{ }^{19}$ Although clinical reasoning has been studied for many years, it only appeared in the competency or learning outcome descriptions after $2000 .{ }^{20-22}$ Therefore, we decided to purposively sample the articles from 2000 onwards. Articles are included, based on the topic (clinical reasoning, clinical judg(e)ment, decision making (if related to the 'how' (descriptive) of reasoning), on the population (registered practising nurses and physicians), with a focus on nursing and medical practice. Empirical (qualitative and quantitative) studies were included, as well as theoretical articles (literature reviews, scoping and systematic reviews, concept analysis and expert opinions). Articles about professional development and education, critical thinking, normative decision-making strategies, case and disease descriptions, books and dissertations are excluded, since these documents are not deemed relevant for our research question. All articles are transferred to Rayyan, to support screening and selection (JCV, RAK). A Preferred Reporting Items for Systematic Reviews and Meta-Analyses flow chart will be used to summarise the selection process of studies, eligible for further analysis and synthesis.

\section{Data evaluation (to be commenced)}

To appraise the quality of included studies which are possibly methodologically diverse, we adapted the instrument developed for integrative reviews by Badu et al, ${ }^{23}$ by adding the criteria for text and opinions of Joanna Briggs Institute. ${ }^{24} \mathrm{~A}$ quality assessment is recommended by Toronto and Remington. ${ }^{15}$ For every type of study, the corresponding screening questions will be evaluated. Two reviewers will independently assess methodological quality. Articles are included in the next phase if they address the research question, and if the score according to their design is higher than $50 \%$, expressing medium to high quality.

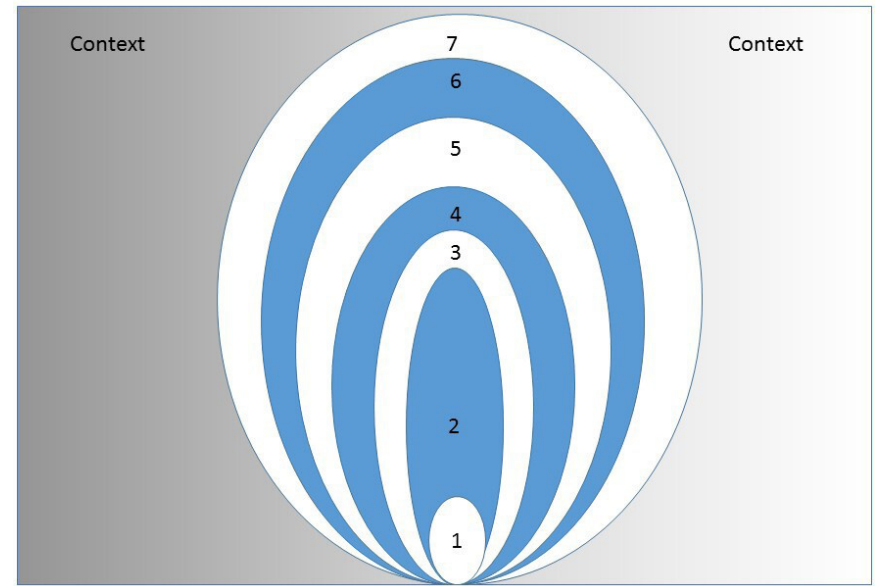

Figure 2 Layers of concept analysis. 1=professional paradigm; $2=$ theories; 3 =intentions; $4=$ =antecedents; $5=$ attributes; $6=$ outcomes; $7=$ content.

We refer to online supplemental appendix 2 for the critical appraisal instrument.

\section{Data analysis}

This phase consists of data extraction (categorising and summarising), data display, comparison and synthesis. Clinical reasoning can be studied from many angles, for example, reasoning strategies, outcomes, skills or context. $^{5}$ Inspired by concept analysis of Walker and Avant ${ }^{14}$ and layered analysis of an educational intervention of Cianciolo and Regehr ${ }^{13}$ and Chamberland et al, ${ }^{25}$ we developed a data extraction form to investigate all layers, such as, content, attributes and perspectives of reasoning. These two approaches were merged and are depicted in an 'onion diagram' in figure 2. The onion is used more often as a metaphor when examining constructs. ${ }^{26}$

We propose to use this multilayered approach to organise and categorise the extensive and diverse data on clinical reasoning of the included studies. The data will be extracted, independently by two reviewers. Our aim with this phase is to get a grip on the data, and to do justice to the rich research on clinical reasoning. The provisional definition of the layers is described in table 1 .

The layers will be further described in the review report.

Our last step is inspired by Cottrell, ${ }^{27}$ who described simultaneous concept analysis. We plan to present our findings in a 'validity matrix', with a column for nursing and medicine, to make the findings suitable for comparing and contrasting.

\section{Data presentation}

The data of previous stages will be presented in tables and figures.

All data will be available on request from the first author.

\section{Ethics and dissemination}

This study involves no human participants and is based on published studies. As such, ethical approval is not required. 


\begin{tabular}{|c|c|c|}
\hline 1 & $\begin{array}{l}\text { Professional } \\
\text { paradigm }\end{array}$ & $\begin{array}{l}\text { A constellation of shared beliefs, } \\
\text { agreements, habits, language and } \\
\text { procedures }^{2829}\end{array}$ \\
\hline 2 & Theories & $\begin{array}{l}\text { The use of descriptive theories to } \\
\text { underpin clinical reasoning }\end{array}$ \\
\hline 3 & Intentions & Reported goals and intentions \\
\hline 4 & Antecedents & $\begin{array}{l}\text { Events, phenomena, behaviours, } \\
\text { conditions or attitudes that occur } \\
\text { prior }^{14}\end{array}$ \\
\hline 5. & Attributes & Characteristics $^{14}$ and techniques ${ }^{13}$ \\
\hline 6. & Outcomes & Results of clinical reasoning \\
\hline 7. & Content & The domain and 'what' \\
\hline & $\begin{array}{l}\text { Contextual } \\
\text { factors }\end{array}$ & The influences from environment \\
\hline
\end{tabular}

Our findings will be disseminated through a publication and through presentations on conferences and will lead to the choices for further studies in the PhD track of the first author (JCV).

\section{Implications}

To our knowledge, this integrative review on clinical reasoning in the medical and nursing profession will be the first systematic study to compare and contrast reasoning of both professions in order to create conceptual clarity. This clarity is needed to optimise interdisciplinary collaboration in patient care, for considering the transferability of study results in another professional domain, and for educators to design training and to guide their students. The used method to extract and analyse data is new and might inspire other researchers on other complex topics.

\section{Twitter Rashmi A Kusurkar @r kusurkar}

Contributors JCV and RAK are responsible for the initial design of this study. HK and JCV developed and executed the systematic search strategy. JCV and RAK selected eligible studies, and SS and JCV will run the quality assessment and will execute the data extraction. JCV conceptualised the review approach, the quality assessment and data extraction, and wrote the first and final drafts of the protocol. JD, EJFMC, MER and RAK contributed to the conceptualisation of the methodology, and to the writing, reviewing and editing of the final protocol.

Funding The authors have not declared a specific grant for this research from any funding agency in the public, commercial or not-for-profit sectors.

Competing interests None declared.

Patient consent for publication Not required.

Provenance and peer review Not commissioned; externally peer reviewed.

Supplemental material This content has been supplied by the author(s). It has not been vetted by BMJ Publishing Group Limited (BMJ) and may not have been peer-reviewed. Any opinions or recommendations discussed are solely those of the author(s) and are not endorsed by BMJ. BMJ disclaims all liability and responsibility arising from any reliance placed on the content. Where the content includes any translated material, BMJ does not warrant the accuracy and reliability of the translations (including but not limited to local regulations, clinical guidelines, terminology, drug names and drug dosages), and is not responsible for any error and/or omissions arising from translation and adaptation or otherwise.

Open access This is an open access article distributed in accordance with the Creative Commons Attribution Non Commercial (CC BY-NC 4.0) license, which permits others to distribute, remix, adapt, build upon this work non-commercially, and license their derivative works on different terms, provided the original work is properly cited, appropriate credit is given, any changes made indicated, and the use is non-commercial. See: http://creativecommons.org/licenses/by-nc/4.0/.

ORCID iD

Jettie Vreugdenhil http://orcid.org/0000-0002-3063-7657

\section{REFERENCES}

1 Young ME, Thomas A, Lubarsky S, et al. Mapping clinical reasoning literature across the health professions: a scoping review. BMC Med Educ 2020;20:107.

2 Spaink K. Singel Uitgeverijen. In: Vallende vrouw. Autobiografie van een lichaam, 2009.

3 Pottier P, Planchon B. [Description of the mental processes occurring during clinical reasoning]. Rev Med Interne 2011;32): :383-90.

4 Yazdani S, Hoseini Abardeh M. Five decades of research and theorization on clinical reasoning: a critical review. Adv Med Educ Pract 2019;10: :703-16.

5 Young M, Thomas A, Gordon D, et al. The terminology of clinical reasoning in health professions education: implications and considerations. Med Teach 2019;41:1277-84.

6 Victor-Chmil J. Critical thinking versus clinical reasoning versus clinical judgment: differential diagnosis. Nurse Educ 2013;38:34-6.

7 Holder AG. Clinical Reasoning: a state of the science report. Int $J$ Nurs Educ Scholarsh 2018;15:1-10.

8 Brown Tyo M, McCurry MK. An integrative review of clinical reasoning teaching strategies and outcome evaluation in nursing education. Nurs Educ Perspect 2019;40:11-17.

9 Muller-Juge V, Cullati S, Blondon KS, et al. Interprofessional collaboration on an internal medicine ward: role perceptions and expectations among nurses and residents. PLOS One 2013;8:e57570.

10 Visser CLF, Kusurkar RA, Croiset G, et al. Students' motivation for interprofessional collaboration after their experience on an IPE ward: a qualitative analysis framed by self-determination theory. Med Teach 2019;41:44-52.

11 Koufidis C, Manninen K, Nieminen J, et al. Unravelling the polyphony in clinical reasoning research in medical education. J Eval Clin Pract 2021;27:438-50.

12 Chiffi D, Zanotti R. Medical and nursing diagnoses: a critical comparison. J Eval Clin Pract 2015;21:1-6.

13 Cianciolo AT, Regehr G, Theory L. Learning theory and educational intervention: producing meaningful evidence of impact through layered analysis. Acad Med 2019;94:789-94.

14 Walker LO, Avant KC. Strategies for theory construction in nursing. Edinburgh Gate: Pearson, 2014.

15 Toronto CE, Remington R. A step-by-step guide to conducting an integrative review. Springer, 2020.

16 Whittemore R, Knafl K. The integrative review: updated methodology J Adv Nurs 2005;52:546-53.

17 Kirkevold M. Integrative nursing research - an important strategy to further the development of nursing science and nursing practice. $J$ Adv Nurs 1997;25:977-84.

18 Poirier T, Behnen $\mathrm{E}$. Where and how to search for evidence in the education literature: the wheel. Am J Pharm Educ 2014;78:70-8.

19 Ames $\mathrm{H}$, Glenton $\mathrm{C}$, Lewin S. Purposive sampling in a qualitative evidence synthesis: a worked example from a synthesis on parental perceptions of vaccination communication. BMC Med Res Methodol 2019;19:26

20 Shumway JM, Harden RM, Association for Medical Education in Europe. AMEE guide No. 25: the assessment of learning outcomes for the competent and reflective physician. Med Teach 2003;25:569-84.

21 Frank J. A history of CanMEDS; the canmeds project: the royal college of physicians and surgeons of Canada moves medcial education into the 21st century. Royal College of Physicians \& Surgeons of Canada 75th Anniversary, Ottawa, Canada, 2004.

22 Bechtel GA, Davidhizar R, Bradshaw MJ. Problem-based learning in a competency-based world. Nurse Educ Today 1999;19:182-7.

23 Badu E, O'Brien AP, Mitchell R. An integrative review of potential enablers and barriers to accessing mental health services in Ghana. Health Res Policy Syst 2018;16:110.

24 Joanna-Briggs-Institute. Joanna briggs institute reviewer's manual, 2017.

25 Chamberland M, Mamede S, Bergeron L, et al. A layered analysis of self-explanation and structured reflection to support clinical reasoning in medical students. Perspect Med Educ 2021;10:171-9.

26 Asher D, Popper M. Tacit knowledge as a multilayer phenomenon: the "onion" model. The Learning Organization 2019;26:264-75. 
27 Cottrell L. Joy and happiness: a simultaneous and evolutionary concept analysis. J Adv Nurs 2016;72:1506-17.

28 Franco AA, Bouma H, Bronswijk JEMHV. Health care paradigms in transition. Gerontechnology 2014;13.
29 Varpio L, MacLeod A. Philosophy of science series: harnessing the multidisciplinary edge effect by exploring paradigms, ontologies, epistemologies, axiologies, and methodologies. Acad Med 2020;95:686-9. 